\title{
Optimal current reference calculation for MMCs considering converter limitations
}

\author{
Daniel Westerman Spier, Graduate Student Member, IEEE, Eduardo Prieto-Araujo, Member, IEEE, \\ Joaquim López-Mestre and Oriol Gomis-Bellmunt, Senior Member, IEEE
}

\begin{abstract}
The paper addresses an optimization-based reference calculation method for Modular Multilevel Converters (MMC) operating in normal and constrained situations (when the converter needs to prioritize its quantities as it has reached voltage or current limitations, e.g. during system faults). The optimization problem prioritizes to satisfy the external AC active and reactive current set-points demanded by the grid operator through the corresponding grid code. If the operator demands are fulfilled, it uses the available MMC degrees of freedom to minimize the arm inductance losses. Otherwise, if the operator demanded AC setpoints cannot be accomplished, the optimization attempts to minimize the error prioritizing between either AC active or reactive currents. The optimization problem constraints are imposed through a steady-state model considering simultaneously the external and internal AC and DC magnitudes of the converter. The steady-state model also includes the voltage variation in the equivalent arm capacitors (considering the ripple). Then, the imposed limitations are the maximum allowed grid and arm currents, the maximum allowed arm voltages and the sub-module capacitor maximum voltages. The paper presents a detailed formulation of the optimization problem and applies it to several case studies where it is shown that the presented approach can be potentially used to obtain the MMC references both in normal and fault conditions.
\end{abstract}

\section{Index Terms}

Modular multilevel converter (MMC), grid support, reference optimization, steady-state analysis.

\section{INTRODUCTION}

$\mathbf{M}$ odular multilevel converters have become the preferred choice for modern High Voltage DC (HVDC) transmission systems [1]-[3]. Compared to classic two-level Voltage Source Converters (VSC), MMCs present easier scalability to higher voltages, improved efficiency due to lower switching frequencies and higher output voltage quality [4]-[6]. MMCs also have additional degrees of freedom that can be used for an improved converter performance [7], specially during AC and DC network imbalances.

In order to adequately exploit these degrees of freedom, the important complexity of the MMC requires models to provide full comprehension of its working principles and the distinct roles of its inner quantities. Relevant previous works on the MMC analysis have been done for both normal and fault scenarios. For normal operation, distinct studies have been performed to analyze the MMC steady-state and dynamic behavior to design the passive elements of the converter [8]-[11]. For unbalanced AC faults, different analysis have also been developed. In [12], the steady-state model of the MMC is derived in the synchronous $d q 0$ frame considering positive and negative sequence components, focusing on the AC side quantities. An alternative analysis method is to derive the steady-state equations in the $a b c$ additive $x^{\Sigma}$ and differential $x^{\Delta}$ frame [13]-[16], which gives additional appreciation on the converter analysis. In [13], a model of the MMC in the $a b c$ frame is proposed for unbalanced grid conditions in order to suppress the injection of $\mathrm{AC}$ current components into the DC side of the converter. However, the characteristics of the AC network during the fault are based only on the positive sequence. To provide insights about the interactions between the positive and negative sequence components of the AC grid, [14] and [15] used both components during the model derivation. Also, in [16], an optimization algorithm based on Lagrange multipliers is proposed to calculate the MMC circulating current considering the positive, negative and zero sequence components of the AC side of the converter.

The aforementioned models, although accurately represent the MMC converter, might not be straightforwardly usable in an optimization environment where constraints in the natural $a b c$ frame should be imposed to individual arm quantities. A natural $a b c$ per arm modelling approach provides a direct identification of the quantities of the converter. Based on this approach, references [17], [18] proposed a steady-state analysis of the MMC focusing on the $\mathrm{AC}$ arm quantities, while considering per phase model of the DC ones, without analyzing DC unbalances.

In terms of converter operation, during unbalanced faults the MMC control should be able to prioritize the AC current components (active or reactive) to be injected into the network to meet the requirements imposed by the grid codes. For

Daniel Westerman Spier and Joaquim López-Mestre are with Control Intel-ligent de l'Energia, coop, 08310, Argentona, Barcelona, Spain. E-mails: daniel.westerman, quimlopez@ cinergia.coop.

Eduardo Prieto-Araujo and Oriol Gomis-Bellmunt are with the Centre d'Innovació Tecnològica en Convertidors Estàtics i Accionaments, Departament d’Enginyeria Elèctrica, Universitat Politècnica de Catalunya, Barcelona 08028, Spain. E-mails: eduardo.prieto-araujo, oriol.gomis@upc.edu. E. Prieto-Araujo is also a Serra Húnter Lecturer. O. Gomis-Bellmunt is also an ICREA Academia researcher.

This project has received funding from the European Union's Horizon 2020 research and innovation programme under the Marie Sklodowska-Curie grant agreement no. 765585. This document reflects only the author's views; the European Commission is not responsible for any use that may be made of the information it contains. This work has also been partially funded by FEDER/Ministerio de Ciencia, Innovación y Universidades - Agencia Estatal de Investigación, Project RTI2018-095429-B-I00 and by the ICREA Academia program. 
instance, reference [19] prioritizes the active current components, whereas [20], [21] main concern is the injection of reactive current to provide voltage support during the fault. In addition, these conditions should be met without violating any of the voltage and current limitations of the converter.

To the best of the authors knowledge, a general optimization problem to calculate the converter references, using a complete per arm MMC model running on the natural $a b c$ reference frame, able to meet the grid operator specifications while considering the constraints of the MMC (arm currents, voltages and sub-module voltages) for any grid AC and DC voltage condition, has not been proposed yet. Next, the main contributions of the article are detailed:

- Development of a natural $a b c$ reference frame steady-state model including all the MMC degrees of freedom.

- Formulation of an optimization-based reference calculation problem using the steady-state developed model, to guarantee an optimal MMC operation under any network voltage conditions and grid operator requirements, considering the converter limitations.

The suggested reference calculation optimization problem ensures that:

- The AC currents are as close as possible to the grid code requirements, without exceeding the MMC limits.

- The internal currents of the converter are limited per arm simultaneously considering both AC and DC components.

- Converter applied voltages do not exceed the limitations of the MMC arms.

- Sub-modules voltages do not exceed their voltage limitation. This constraint is imposed using an equivalent arm capacitor modelling which includes the corresponding voltage fluctuation (assuming that an adequate sorting algorithm is implemented).

- The degrees of freedom of the MMC are fully exploited by the optimization to be as close as possible to the operator requirements.

- Any AC grid operator requirement can be fulfilled, prioritizing either active or reactive current component. In this paper, the reactive $\mathrm{AC}$ current is prioritized in order to provide voltage support to the faulted network.

- It can be used for different MMC sub-module configurations (half-bridge, full-bridge, hybrid), as long as the MMC limits are adapted according to each equivalent arm voltage condition.

To validate the proposed optimization-based reference calculation, different case studies are included to show the optimization performance and to highlight the effect of the imposed constraints. In addition, the output values from the steady-state model are compared with time domain simulations (once steady-state is reached) for different grid voltage conditions, showing adequate results.

\section{MMC MODEL DESCRIPTION}

The three-phase MMC (see Fig. 1) is a VSC consisting of three legs, one per phase, in which each leg has two stacks of $N_{\text {arm }}$ sub-modules, known as the upper and lower arms. The sub-modules can vary from simple topologies such as the half-bridge or full-bridge to complex ones, based on the application requirements [22]. The main quantities for each phase, considering $k \in(a, b, c)$, are the AC grid voltages $u_{g}^{k}$, the voltage at the neutral point $u_{n}$, the upper and lower arms voltages $u_{u}^{k}$ and $u_{l}^{k}$, the upper and lower DC grid voltages $U_{u}^{D C}$ and $U_{l}^{D C}$, the upper and lower arm currents $i_{u}^{k}$ and $i_{l}^{k}$ and the AC grid current $i_{s}^{k}$. $R_{a}$ and $L_{a}$ are the arm equivalent resistance and inductance and finally, $R_{s}$ and $L_{s}$ are the phase reactor equivalent resistance and inductance. The voltage equations per phase can be written as

$$
\begin{aligned}
U_{u}^{D C}-u_{u}^{k}-u_{g}^{k}-u_{n} & =R_{a} i_{u}^{k}+L_{a} \frac{d i_{u}^{k}}{d t}+R_{s} i_{s}^{k}+L_{s} \frac{d i_{s}^{k}}{d t} \\
-U_{l}^{D C}+u_{l}^{k}-u_{g}^{k}-u_{n} & =-R_{a} i_{l}^{k}-L_{a} \frac{d i_{l}^{k}}{d t}+R_{s} i_{s}^{k}+L_{s} \frac{d i_{s}^{k}}{d t}
\end{aligned}
$$

Combining the static form of the previous equations and the converter power flow equations, it is possible to derive a steady-state model of the converter in the $a b c$ reference frame, detailed in Section III. Then, this model will be adapted to be included in the optimization problem formulation as explained in Section V.

\section{STEADY-STATE MODELLING AND ANALYSIS}

In this section, the steady-state equations of the MMC are derived considering that it is connected to generic $\mathrm{AC}$ and $\mathrm{DC}$ networks, which can be either in balanced or unbalanced conditions. The MMC arm quantities contain both AC and DC components. Applying the superposition principle [23], the DC and AC systems can be decoupled and studied separately.

\section{A. AC network currents}

For steady-state analysis purposes, the phasorial notation $\underline{X}^{k}=X_{r}^{k}+j X_{i}^{k}=X^{k} \angle \theta^{k}$ will be adopted, with $x(t)=$ $X^{k} \mathbb{R}\left(e^{j\left(\omega t+\theta^{k}\right)}\right)$ and $k \in(a, b, c)$. The model assumes that the active $P^{k}$ and reactive $Q^{k}$ power magnitudes and per phase voltages $\underline{U}_{g}^{k}$ are given, as the MMC connection with the AC network considers a grid-following control mode. Based on these values, the grid currents $\underline{I}_{s}^{k}$ can be obtained 


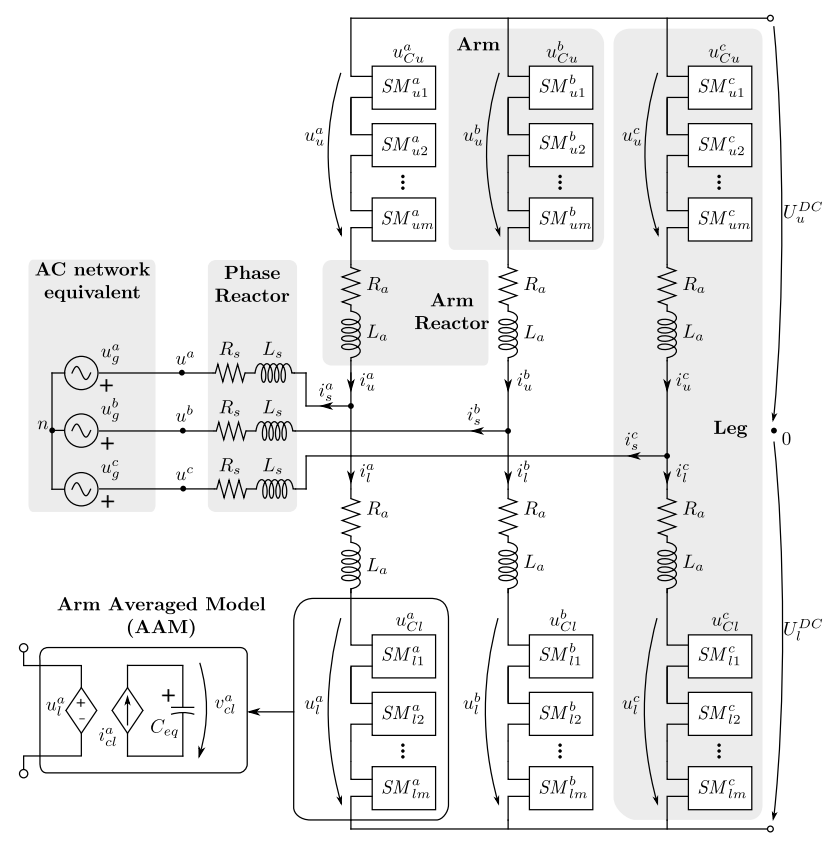

Fig. 1. Complete model of the MMC converter.

$$
\underline{I}_{s}^{k}=\left(\frac{\underline{S}^{k}}{\underline{U}_{g}^{k}}\right)^{*}=\frac{P^{k}-j Q^{k}}{\underline{U}_{g}^{k *}}
$$

Using (3), the calculation of $\underline{I}_{s}^{k}$ may result in zero sequence components $\underline{I}_{s}^{0}$ for a generic unbalanced AC voltage condition, which cannot flow due to the three-wire $\mathrm{AC}$ connection ${ }^{1}$. To impose this condition in the model, one option is to remove the zero-sequence keeping the positive and negative sequence components [24]. Current $\underline{I}_{s}^{k}$ will be subsequently used in the following section as an input to solve the steady-state model.

\section{B. AC MMC circuit analysis}

Fig. 2 shows the AC circuit which can be derived from Fig. 1, short-circuiting the DC voltage sources and considering the AC component of the arm applied voltages.

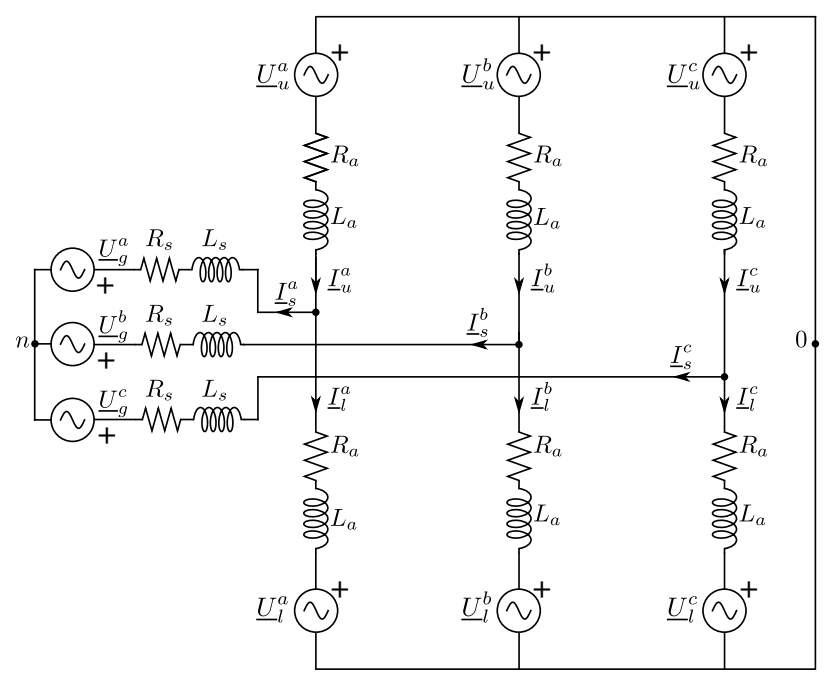

Fig. 2. AC model of the MMC.

\footnotetext{
${ }^{1}$ Note that the AC current reference calculation (3) would not be used in a real converter operation, in which grid code requirements would impose the AC current set-point values based on the defined grid conditions, as it is detailed in Sections IV and V. In this case, equation (3) is included in this section so that the analysis stands on its own, being an input for the model.
} 
Applying the Kirchhoff Current Law (KCL) in the middle point of the MMC arms and involving the currents calculated in (3), the relation between the arm currents is obtained as

$$
\underline{I}_{s}^{k}=\underline{I}_{u}^{k}-\underline{I}_{l}^{k}
$$

To prevent the circulation of AC current through the DC network, no AC zero sequence arms' current component must be flowing through the MMC. This restriction can be met by imposing that the sum of the upper arms' AC currents is equal to zero, as

$$
\underline{I}_{u}^{a}+\underline{I}_{u}^{b}+\underline{I}_{u}^{c}=0
$$

Note that, no additional equation is required to eliminate the zero sequence current component from the lower arms, as it can be directly obtained by combining (4), (5) while assuming no zero sequence in the grid currents (three-wire connection).

The AC voltage equations for the MMC arms (see Fig. 2) can be obtained through Kirchhoff Voltage Law (KVL), as

$$
\begin{aligned}
& \underline{U}_{0 n}=\underline{U}_{g}^{k}+\underline{Z}_{s}\left(\underline{I}_{u}^{k}-\underline{I}_{l}^{k}\right)+\underline{Z}_{a} \underline{I}_{u}^{k}+\underline{U}_{u}^{k} \\
& \underline{U}_{0 n}=\underline{U}_{g}^{k}+\underline{Z}_{s}\left(\underline{I}_{u}^{k}-\underline{I}_{l}^{k}\right)-\underline{Z}_{a} \underline{I}_{l}^{k}-\underline{U}_{l}^{k}
\end{aligned}
$$

where, $\underline{U}_{0 n}$ is the voltage between the 0 DC reference node and the neutral $n$ of the AC three-phase system and, $\underline{Z}_{s}$ and $\underline{Z}_{a}$ are the phase reactor and MMC's arm impedances, respectively.

The last equation to be included in the $\mathrm{AC}$ analysis is the power difference between the arms, which is required to conduct a first steady-state analysis. In this case, it can be assumed that the upper and lower arms exchange the same amount of active and reactive power, as

$$
\begin{aligned}
& P_{u}^{k}-P_{l}^{k}=0 \rightarrow U_{u_{r}}^{k} I_{u_{r}}^{k}+U_{u_{i}}^{k} I_{u_{i}}^{k}=U_{l_{r}}^{k} I_{l_{r}}^{k}+U_{l_{i}}^{k} I_{l_{i}}^{k} \\
& Q_{u}^{k}-Q_{l}^{k}=0 \rightarrow U_{u_{r}}^{k} I_{u_{i}}^{k}-U_{u_{i}}^{k} I_{u_{r}}^{k}=U_{l_{r}}^{k} I_{l_{i}}^{k}-U_{l_{i}}^{k} I_{l_{r}}^{k}
\end{aligned}
$$

with $P_{u, l}^{k}$ and $Q_{u, l}^{k}$ as the upper and lower arms active and reactive power, respectively. Later, (8) and (9) will not be part of the optimization, as they will be considered a degree of freedom of the converter, as discussed in Section V.

\section{DC MMC circuit analysis}

For the DC part of the MMC, an analogous analysis to the AC circuit is developed. The DC model is shown in Fig. 3, where only DC voltages are applied to the system, short-circuiting the AC sources and inductances.

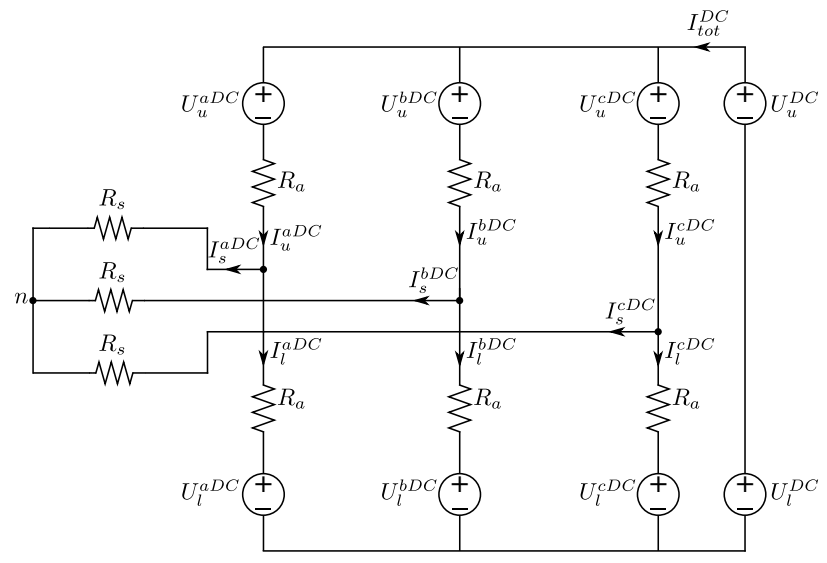

Fig. 3. DC model of the MMC.

In order to avoid the circulation of DC components into the AC network, $I_{s}^{k D C}$ is regulated to be equal to zero. Therefore, $I_{u}^{k D C}=I_{l}^{k D C}=I^{k D C}$, with $k \in(a, b, c)$. The voltage equations can be written as follows

$$
U_{u}^{D C}+U_{l}^{D C}=U_{u}^{k D C}+U_{l}^{k D C}+2 R_{a} I^{k D C}
$$

Also, the total DC current of the system $I_{\text {tot }}^{D C}$ can be obtained as

$$
I_{t o t}^{D C}=I^{a D C}+I^{b D C}+I^{c D C}
$$




\section{Steady-state AC/DC power balance equations}

In steady-state conditions, the AC and DC active power exchanged in each of the arms (voltage sources) should be equal, if the semi-conductor losses are neglected. Obviously, this is not feasible with instantaneous values (as the arm single-phase AC power is non-constant). However, it is possible to impose an equality between the AC average power (calculated in the phasor domain) and the DC power. If this condition is not achieved, the energy in the arm cells would either charge or discharge the equivalent arm capacitors (increasing/decreasing the stored energy) and therefore, steady-state conditions would not hold. This constraint can be mathematically expressed as

$$
P_{u, l}^{k A C}=P_{u, l}^{k D C} \rightarrow U_{u, l_{r}}^{k} I_{u, l_{r}}^{k}+U_{u, l_{i}}^{k} I_{u, l_{i}}^{k}=U_{u, l}^{k D C} I^{k D C}
$$

\section{E. Equivalent arm capacitor voltage fluctuation}

Next, an estimation of the equivalent arm capacitor (see Fig. 1) voltage ripple is performed in order to verify that the sub-module capacitor voltages do not exceed their design limitations. The energy stored in each arm of the converter is directly dependable on the power flowing through the MMC. Under balanced AC network conditions, the average energy stored in each arm is equal, also showing similar ripple profiles per arm (phase-shift difference). However, during unbalanced scenarios, the MMC arms may present high energy deviations, leading to variations of the energy stored within them, which might eventually exceed the design limitations. Consequently, the energy stored in each arm, therefore in each sub-module (assuming an adequate sorting algorithm), must be kept within a certain range to ensure a proper operation of the MMC.

To mathematically represent the equivalent arm voltage fluctuation, the Arm Averaged Model (AAM) [25] is used (see Fig. 1). This model represents each arm as an equivalent capacitor that is charged/discharged depending on the power exchanged by each arm, which is reflected as a charging/discharging current. This way, the entire arm can be represented as an equivalent capacitor. Based on the AAM equivalent arm model, a mathematical procedure to derive a generalized estimation for the maximum and minimum voltage ripple for the equivalent arm capacitor is presented.

1) Instantaneous MMC arm power: To calculate the equivalent arm capacitor voltage ripple, firstly, the instantaneous power for the upper and lower arms of the MMC should be obtained as

$$
\begin{gathered}
p_{u, l}^{k}(t)=u_{u, l}^{k}(t) i_{u, l}^{k}(t)=\left(U_{u, l}^{k D C}+\hat{U}_{u, l}^{k} \cos \left(\omega t+\psi_{u, l}^{k}\right)\right) \\
\left(I_{u, l}^{k D C}+\hat{I}_{u, l}^{k} \cos \left(\omega t+\delta_{u}^{k}\right)\right)
\end{gathered}
$$

where $I_{u, l}^{k D C}$ are the upper and lower DC currents flowing through the MMC arms, $\delta_{u, l}^{k}$ and $\psi_{u, l}^{k}$ are the upper and lower arms' currents and voltages phase-angles, respectively. It can be noted that the power will have components at zero, $\omega$ and $2 \omega$ pulsations. The sum of the constant zero frequency power of the AC and DC sources must be zero in steady-state conditions, to avoid energy deviation in the arm. Therefore, the upper and lower arms' powers [26] can be reduced to

$$
\begin{gathered}
p_{u, l}^{k}(t)=U_{u, l}^{k D C} \hat{I}_{u, l}^{k} \cos \left(\omega t+\delta_{u, l}^{k}\right)+ \\
+I_{u, l}^{k D C} \hat{U}_{u, l}^{k} \cos \left(\omega t+\psi_{u, l}^{k}\right)+\frac{\hat{U}_{u, l}^{k} \hat{I}_{u, l}^{k}}{2} \cos \left(2 \omega t+\delta_{u, l}^{k}+\psi_{u, l}^{k}\right)
\end{gathered}
$$

2) Arms maximum energy and voltage calculation: The voltage in a capacitor presents a profile that is related to its energy. One way to find the maximum and minimum values of the equivalent arm capacitor voltage is to obtain the peak values of the arm energy. The equation for the instantaneous energy flowing through the MMC arms can be expressed as (integrating (14) over time)

$$
\begin{gathered}
E_{u, l}^{k}(t)=\frac{U_{u, l}^{k D C} \hat{I}_{u, l}^{k}}{\omega} \sin \left(\omega t+\delta_{u, l}^{k}\right)+ \\
+\frac{I_{u, l}^{k D C} \hat{U}_{u, l}^{k}}{\omega} \sin \left(\omega t+\psi_{u, l}^{k}\right)+\frac{\hat{U}_{u, l}^{k} \hat{I}_{u, l}^{k}}{4 \omega} \sin \left(2 \omega t+\delta_{u, l}^{k}+\psi_{u, l}^{k}\right)
\end{gathered}
$$

The upper and lower arm energies, shown in (15), have terms that oscillate at the fundamental frequency and a second order one. As stated in the comprehensive analysis developed in [27], the actual maximum energy stored in the equivalent arm capacitor, and consequently its maximum voltage ripple, cannot be found without interactive methods. However, as demonstrated in [26], it is possible to calculate the absolute maximum energy bound for the MMC's upper and lower arms $E_{u, l_{\max }}^{k A C}$, as shown in (16). This absolute maximum energy value will result in higher energy levels than the actual magnitude for scenarios where $\left(\delta_{u, l}^{k}+\psi_{u, l}^{k} \neq 90^{\circ}\right)$; thus, it can be used as a safety value to be employed in the formulation of the optimization problem. Considering steady-state conditions, the MMC equivalent arms' capacitors are storing the nominal DC energy, obtained as

$$
E_{u, l_{\text {ref }}}^{k}=\frac{C_{S M}}{2} N_{u, l_{\text {arm }}}^{k} U_{S M}^{2}
$$




$$
E_{u, l_{\text {max }}}^{k A C} \approx \sqrt{\left[\frac{U_{u, l}^{k D C} \hat{I}_{u, l}^{k}}{\omega} \cos \left(\delta_{u, l}^{k}\right)+\frac{I_{u, l}^{k D C} \hat{U}_{u, l}^{k}}{\omega} \cos \left(\psi_{u, l}^{k}\right)\right]^{2}+\left[\frac{U_{u, l}^{k D C} \hat{I}_{u, l}^{k}}{\omega} \sin \left(\delta_{u, l}^{k}\right)+\frac{I_{u, l}^{k D C} \hat{U}_{u, l}^{k}}{\omega} \sin \left(\psi_{u, l}^{k}\right)\right]^{2}}+\left|\frac{\hat{U}_{u, l}^{k} \hat{I}_{u, l}^{k}}{4 \omega}\right|
$$

where $U_{S M}$ is average sub-module nominal voltage, $C_{S M}$ is the sub-module capacitance and $N_{u, l_{a r m}}^{k}$ is the number of submodules available in the arm. Therefore, the maximum and minimum values for the upper and lower arms energy can be obtained adding (16) and (17), described as

$$
E_{u, l_{\text {max }, \text { min }}}^{k}=\underbrace{E_{u, l_{\text {ref }}}^{k}}_{\text {DC term (17) }} \pm \underbrace{E_{u, l_{\text {max }}}^{k A C}}_{\text {peak of the AC part (16) }}
$$

Even though several operating conditions will result in equivalent arm capacitor energy fluctuations that are asymmetric regarding their DC component, the maximum and minimum energy bounds for any operating point will always present a symmetric pattern (since they are derived considering the highest energy ripple scenario). Furthermore, (18) will be used in the formulation of the optimization problem to avoid not only over-modulations but also exceeding equivalent arm capacitors and SM capacitors over-voltages in the MMC, as will be shown in Section V and VI. Based on the maximum and minimum energy levels $E_{u, l_{\max , \min }}^{k}$, the admissible voltage magnitudes of the equivalent arms' capacitors can be expressed as

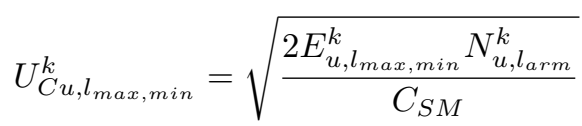

3) Complete model: The full non-linear steady-state model for the MMC, with $k \in(a, b, c)$, can be summarized as,

- 72 quantities given as:

- 13 complex AC quantities: $\underline{U}_{u, l}^{k}, \underline{U}_{0 n}, \underline{I}_{u, l}^{k}$

- 46 DC quantities: $U_{u, l}^{k D C}, I^{k D C}, I_{t o t}^{D C}, E_{u, l_{\max }}^{k A C}, E_{u, l_{r e f}}^{k} E_{u, l_{\max , \min }}^{k}, U_{C u, l_{\max , \min }}^{k}$

- 72 equations divided as:

- 10 complex linear AC equations: (4) to (7)

- 4 linear DC real equations: (10), (11)

- 48 non-linear real equations: (8), (9), (12), (16-19)

The model will require the following inputs

- AC and DC system voltages: $\underline{U}_{g}^{k}, U_{u, l}^{D C}$.

- AC network current $\underline{I}_{s}^{k}$ obtained using (3), based on $P^{k}, Q^{k}$ and $\underline{U}_{g}^{k}$, eliminating the zero sequence component $\underline{I}_{s}^{0}$.

- Sub-module characteristics: $U_{S M}, C_{S M}, N_{u, l_{\text {arm }}}^{k}$.

This model can be used to calculate the steady-state quantities of the MMC for any grid voltage condition. This paper will adapt it in Section V to use it as part of an optimization-based reference calculation problem. It will also be employed to calculate the pre-fault values of the system, to be added as initial conditions for the optimization algorithm.

\section{GRID SUPPORT REQUIREMENTS}

This section details the calculation of the AC side currents dictated by the operators' grid codes. During an AC grid voltage fault, the MMC must be able to inject or absorb reactive current $\Delta I_{r}$ from the AC grid in order to provide voltage support. According to the Spanish grid code [21] (taken as example), the magnitude of $\Delta I_{r}$ (in pu basis) to be injected into the grid varies according to AC system RMS voltage levels as follows

1) $U_{\min 1} \leqslant U_{g}^{k} \leqslant U_{\max 1} \rightarrow \Delta I_{r}^{k}=0$

2) $U_{\min 2} \leqslant U_{g}^{k}<U_{\min 1} \rightarrow \Delta I_{r}^{k}=\frac{\Delta I_{r \max }\left(U_{\min 1}-U_{g}^{k}\right)}{U_{\min 1}-U_{\min 2}}$

3) $U_{g}^{k}<U_{\min 2} \rightarrow \Delta I_{r}^{k}=\Delta I_{r \max }$

Under a balanced fault, the injection of reactive currents presents a symmetrical profile. However, in an unbalanced AC voltage sag condition, the three-phase system may have different voltage levels in each of its phases [28]. A common solution to provide voltage support is based on the decomposition of the faulted network voltage into positive, negative and zero sequence components. Then, the magnitude of the reactive current to be injected is selected based only on the magnitude of the positive sequence voltage component, which this paper will refer as Strategy I. However, this methodology is unable to provide full voltage support to the faulted grid. Furthermore, by disregarding the negative and zero voltage sequence components, healthy phases might receive unnecessary voltage support leading to over-voltages in the system. The opposite scenario may also happen, where the faulted phases might require full voltage support but, the value of the reactive current calculated based only on the positive sequence voltage component is insufficient. 
Another possible solution would be to consider all the three symmetrical (positive, negative and zero) AC voltages components, which would be the ideal case, as the phases would receive the exact amount of reactive and active power in accordance to the grid code, named Strategy II. Such condition may be infeasible since the AC system consists of three-wires and this strategy might try to impose zero sequence current component into the AC network. For both strategies (I and II), if the magnitude of the AC current references exceeds the AC grid current limitations $I_{\text {max }}^{A C}$, they must be reduced while keeping their phasorial angle constant.

In order to provide a better voltage support, an additional strategy is suggested to adjust each individual phase current to be as close as possible to the grid code (active and specially reactive) power support requests, without violating the converter constraints and limits. Firstly, the pre-fault active and reactive AC grid current components are obtained as

$$
\left[\begin{array}{l}
I_{P_{p r e}}^{k} \\
I_{Q_{p r e}}^{k}
\end{array}\right]=\left[\begin{array}{cc}
\cos \left(\theta_{p r e}^{k}\right) & \sin \left(\theta_{p r e}^{k}\right) \\
-\sin \left(\theta_{p r e}^{k}\right) & \cos \left(\theta_{\text {pre }}^{k}\right)
\end{array}\right] \cdot\left[\begin{array}{c}
I_{\text {spre }_{r}}^{k} \\
I_{\text {spre }_{i}}^{k}
\end{array}\right]
$$

where $\theta_{\text {pre }}^{k}$ and $\underline{I}_{s p r e}^{k}$ are the phase-angles of the AC grid voltages and the AC grid currents during pre-fault conditions (obtained using (3)), respectively. The support current to fulfill the grid code requirements is calculated by adding the additional required reactive current to the pre-fault reactive current component, as $I_{s u p}^{k}=\Delta I_{r}^{k}+I_{Q_{p r e}}^{k}$. This magnitude is expressed as a phasor, which has to be properly placed in relation to the faulted AC grid voltages to allow voltage support, as (considering $k \in(a, b, c))$

$$
\underline{I}_{\text {sup }}^{k}=I_{\text {sup }}^{k}\left(\cos \left(\theta_{F}^{k}+90^{\circ}\right)+j \sin \left(\theta_{F}^{k}+90^{\circ}\right)\right)
$$

where $\theta_{F}^{k}$ is the phase-angle of the AC grid voltages during the fault. Note that the phase-angles during pre-fault and faulted conditions are obtained by means of phase locked-loops (PLLs), which must be fast enough in order to avoid undesirable dynamic interactions with other quantities of the MMC [29]. However, if $\left|I_{\text {sup }}^{k}\right|<I_{\text {max }}^{A C}$, the grid code demands the MMC to also inject active current components $\underline{I}_{P}^{k}$, which must have the same phase-angles as the faulted AC grid voltages, until the AC grid currents achieve nominal levels. This active current component can be described as

$$
\underline{I}_{P}^{k}=I_{P}^{k}\left(\cos \left(\theta_{F}^{k}\right)+j \sin \left(\theta_{F}^{k}\right)\right)
$$

where $I_{P}^{k}$ is the magnitude of the AC active current, which is equal to the pre-fault active current component $I_{P_{p r e}}^{k}$. As an example, for a type $\mathrm{C}$ fault [28], the voltages and current vectors are depicted in Fig. 4. For an easier understanding of the phasors, a pre-fault state with $I_{Q_{p r e}}^{k}=0$ has been chosen.

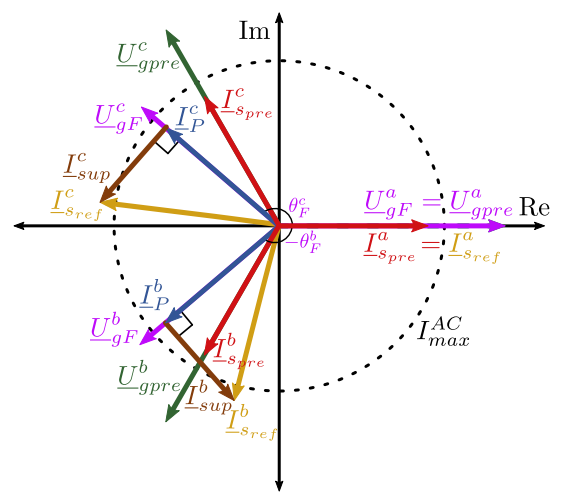

Fig. 4. Reactive current injection according to the utility grid voltage.

Although the addition of $I_{P_{p r e}}^{k}$ may result in AC grid currents that are higher than the nominal levels or even exceeding the system limitations, an optimization problem is formulated to avoid those issues. To do so, the active $I_{P}^{k}$ and reactive $I_{Q}^{k}$ components have to be adjusted to calculate AC grid current references $\underline{I}_{s_{\text {ref }}}^{k}$ that do not exceed the design limitations. This can be achieved by adding to (20) the coefficients $\left(\alpha^{k}\right.$ and $\left.\beta^{k}\right)$, which are used to adapt the amount of active and reactive currents to be injected per phase in case of reaching a limitation. Then, $\underline{I}_{s_{r e f}}^{k}$ can be calculated as

$$
\left[\begin{array}{l}
I_{s_{\text {ref }}}^{k} \\
I_{s_{\text {refi }}}^{k}
\end{array}\right]=\left[\begin{array}{cc}
\cos \left(\theta_{F}^{k}\right) & -\sin \left(\theta_{F}^{k}\right) \\
\sin \left(\theta_{F}^{k}\right) & \cos \left(\theta_{F}^{k}\right)
\end{array}\right] \cdot\left[\begin{array}{c}
\alpha^{k} \cdot I_{P}^{k} \\
\beta^{k} \cdot I_{Q}^{k}
\end{array}\right]
$$

where $I_{Q}^{k}=I_{\text {sup }}^{k}$. As mentioned above, the coefficients $\alpha^{k}$ and $\beta^{k}$ can affect the active and reactive currents levels, respectively. Under normal operations, both parameters present their maximum level being equal to 1, which would not constraint neither the active nor the reactive powers. However, depending on the AC grid voltage conditions, these values should be modified to remove both the zero sequence current component of the unbalanced references and to meet the MMC limitations. Clearly, the 


$$
\operatorname{minimize} \mathrm{W}=\lambda_{1}\left(R_{a}\left(\sum_{k=a}^{c} I_{u}^{k^{2}}+I_{l}^{k^{2}}\right)+R_{a}\left(\sum_{k=a}^{c} I_{u}^{k D C^{2}}+I_{l}^{k D C^{2}}\right)\right)-\lambda_{2}\left(\sum_{k=a}^{c} \alpha^{k}\right)-\lambda_{3}\left(\sum_{k=a}^{c} \beta^{k}\right)
$$

selection of the six coefficients $\alpha^{k}$ and $\beta^{k}$ is not a simple task as changing any of the coefficients will importantly affect the response of the converter towards the network. Thus, the reference calculation problem will be part of an optimization problem, which will ensure that the current references are as close as possible in terms of active and reactive powers to the grid code demands (enabling also active/reactive power prioritization). This strategy is named Optimal Strategy and the optimization problem used to calculate it is detailed in the next section.

\section{Optimal REFERENCE CALCULATION}

In this section, an optimization algorithm is proposed to calculate the converter arm quantities in order to provide adequate grid support during balanced and unbalanced voltage conditions while ensuring that the converter operation is kept within its design and operating limits.

\section{A. Optimization problem}

The multi-objective function is defined in (23), where each term of the expression is multiplied by a weighting factor $\lambda_{x}$ that is used to prioritize among converter arm inductance losses $\lambda_{1}$, active power $\lambda_{2}$ and reactive power $\lambda_{3}$. In this paper, $\lambda_{3} \gg \lambda_{2} \gg \lambda_{1}$ so that the reactive current is prioritized over the active current and the power losses (pushing $\beta^{k}$ as close as possible to 1 ), respectively.

To ensure that all the technical constraints are fulfilled, the optimization problem is subjected to several linear and nonlinear constraints. These mathematical restrictions are based on the equations of the steady-state model presented in Section III. Specifically, (5), (6) and (7), together with the following linear constraints are part of the optimization problem, considering $k \in(a, b, c)$

$$
\begin{gathered}
\underline{I}_{s_{r e f}}^{a}+\underline{I}_{s_{r e f}}^{b}+\underline{I}_{s_{r e f}}^{c}=0 \\
\underline{I}_{s_{r e f}}^{k}=\underline{I}_{u}^{k}-\underline{I}_{l}^{k} \\
U_{u, l}^{D C}=U_{u, l}^{k D C}+R_{a} I_{u, l}^{k D C} \\
I_{s_{r e f}}^{k D C}=I_{u}^{k D C}-I_{l}^{k D C} \\
I_{t o t}^{D C}=I_{u}^{a D C}+I_{u}^{b D C}+I_{u}^{c D C}
\end{gathered}
$$

Equation (25a) imposes that the $\mathrm{AC}$ current references have no zero sequence component, while (25b) describes the relation between the MMC AC currents. Equations (25c) and (25d) are included to guarantee that no DC current flows through the AC side of the converter. Finally, (25e) relates the MMC inner DC currents with the total DC one.

For the non-linear constraints, (24) is needed along with the AC/DC arm power balance equations

$$
P_{u, l}^{k A C}=P_{u, l}^{k D C} \rightarrow U_{u, l_{r}}^{k} I_{u, l_{r}}^{k}+U_{u, l_{i}}^{k} I_{u, l_{i}}^{k}=U_{u, l}^{k D C} I_{u, l}^{k D C}
$$

In addition to the expressions shown above, the energy equations for the upper and lower equivalent arm capacitors (16)-(18) are also required, since they are used to calculate the equivalent arms' capacitor voltages. Lastly, the converter limitations are imposed by the following inequalities

$$
\begin{gathered}
I_{s_{\text {ref }}}^{k} \leqslant I_{\text {max }}^{A C}, \quad I_{u, l}^{k}+I_{u, l}^{k D C} \leqslant I_{\text {max }}^{a r m} \\
U_{C u, l_{\max }}^{k} \leqslant U_{C_{\max }} \\
0 \leqslant U_{u, l}^{k D C}+\sqrt{2} U_{u, l}^{k} \leqslant U_{C u, l_{\min }}^{k} \\
0 \leqslant \alpha^{k} \leqslant 1, \quad 0 \leqslant \beta^{k} \leqslant 1
\end{gathered}
$$

where $I_{\max }^{A C}$ is the maximum current that can be injected into the AC grid, $I_{\max }^{a r m}$ is the maximum current which can circulate through the MMC arms, and $U_{C_{\max }}$ is the peak value that the equivalent arms' capacitors voltage can achieve, that can be directly related to each sub-module voltage. Equation (27a) limits the maximum currents that can be injected into the AC grid and that can flow through the MMC arms. The inequality (27b) ensures that the equivalent arm capacitor voltages do not exceed their maximum voltage bound, whereas $(27 \mathrm{c})$ is used to guarantee that the applied voltages are higher than the minimum 
allowed voltage (left-hand side of the equation) and to avoid over-modulations (right-hand side). If full-bridge sub-modules were considered, the left value of $(27 \mathrm{c})$ should be replaced from 0 to $-U_{C u, l_{m i n}}^{k}$. Finally, the weights $\alpha^{k}$ and $\beta^{k}$ are limited as shown in (27d). The optimization will try to maximize these weights (max. equal to 1), consequently being as close as possible to the desired current references imposed by the grid code, respecting the system constraints.

\section{B. Complete optimization model and methodology}

The complete optimization model is summarized below.

- 90 MMC quantities:

- 16 AC quantities: $\underline{U}_{u, l}^{k}, \underline{U}_{0 n}, \underline{I}_{u, l}^{k}, \underline{I}_{s_{r e}}^{k}$

- 58 DC quantities: $U_{u, l}^{k D C}, I_{u, l}^{k D C}, I_{s_{r e f}}^{k D C}, I_{t o t}^{D C}, \alpha^{k}, \beta^{k}$, $E_{u, l_{\max }}^{k A C}, E_{u, l_{\text {ref }}}^{k}, E_{u, l_{\text {max }, \min }}^{k}, U_{C u, l_{\max , \min }}^{k}$

- 80 equations divided in:

- 11 complex linear AC equations: (5), (6), (7), (25a), (25b)

- 10 linear DC equations: (25c-25e)

- 48 non-linear equations: (16-19), (24), (26)

- 39 inequalities: (27a-27d)

The optimal model will require the following inputs:

- $\mathrm{AC}$ and DC system voltages: $\underline{U}_{g}^{k}, U_{u, l}^{D C}$.

- AC network current in the form of active $I_{P}^{k}$ and reactive $I_{Q}^{k}$ currents, which are obtained using the pre-fault AC current and voltage levels, the reactive current required by the grid code $I_{\text {sup }}^{k}$ and the AC grid phase-angles during the fault $\theta_{F}^{k}$.

- Sub-module type and characteristics: $U_{S M}, C_{S M}, N_{u, l_{a r m}}^{k}$.

The different steps to run the optimization algorithm are:

1) Define the pre-fault and fault condition scenarios.

2) Obtain of the pre-fault MMC steady-state quantities.

3) Calculate the active and reactive current set-points required by the grid code $\underline{I}_{P}^{k}$ and $\underline{I}_{Q}^{k}$, see (20-22).

4) Determine the weighting factors $\lambda_{x}$ of the multi-objective function.

5) Introduce the MMC parameters and limitations.

6) Run the optimization algorithm using equations detailed above.

The optimization algorithm could be implemented following two different strategies as shown in Fig. 5. The first approach is to perform an online optimization, detailed in Fig. 5a, which could be executed using computers, DSPs or FPGAs. The algorithm receives the real-time magnitudes of the HVDC system and it calculates the AC grid, DC and AC arm current references that track the set-points imposed by the TSO while keeping the internal energy of the converter balanced and within the imposed boundaries. Then, conventional MMC inner and grid current controllers could be used to achieve the desired current references.

Another approach would be to run the optimization offline, see Fig. 5b. The optimization is executed offline considering multiple operational points of the converter, saving all the results. These results could be stored as a data table in the memory of a processor unit. Then, whenever this device receives the real-time data from the HVDC system, it is possible to interpolate the desired current control references from the data table which are sent to the different MMC current regulators.

The first configuration ensures that the optimal references are obtained for the specific point of operation, but requires to run the optimization online with the corresponding computational burden. The second option only requires to correlate the actual operating point with the corresponding one in the data table, but it may have some errors depending on the size of the data set up in this table (which might be limited by the memory available in the microcontroller).

\section{Case Study}

In this section, the results of the proposed optimization-based reference calculation algorithm are shown in voltage sag (case A) and constrained (cases B and C) scenarios. The optimization problem is solved using Matlab ${ }^{\circledR}$ fmincon function (interiorpoint based method [30]), since it can deal with constrained nonlinear multi-variable problems. In addition, the optimization results are also applied to a MMC time-domain simulation model developed in Matlab Simulink ${ }^{\circledR}$. The steady-state simulation waveforms and the optimization output are compared to confirm the applicability of the suggested method and to verify that the MMC constrains (currents and voltages limits) are respected. Table I details the system parameters for the case studies.

\section{A. Case A: Optimal reference calculation in AC voltage sags}

This case study is performed to illustrate how the suggested optimal reference calculation is able to provide adequate grid support, compared with the conventional Strategy I and the ideal grid code support Strategy II $^{2}$ (see Section IV). The MMC is

\footnotetext{
${ }^{2}$ Strategy I provides only positive sequence support, based on positive sequence voltage, while Strategy II provides the ideal per phase support following the grid code, including the zero sequence (it is used only as a reference, as it is not implementable).
} 


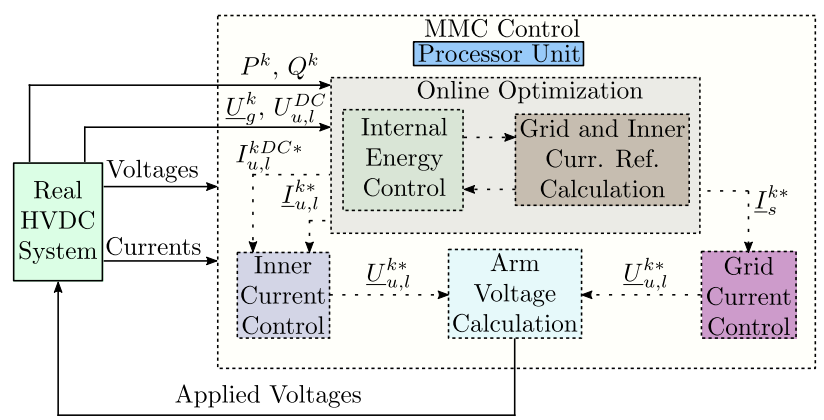

(a) Online optimization.

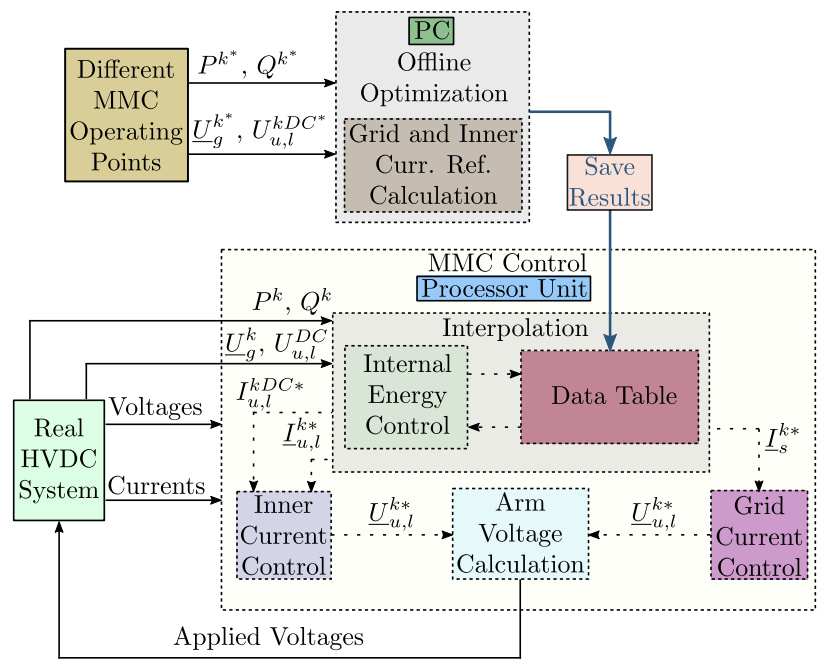

(b) Offline optimization.

Fig. 5. Application scenarios for the proposed optimization algorithm.

TABLE I

SYSTEM PARAMETERS

\begin{tabular}{lccl}
\hline \hline Parameter & Symbol & Value & Units \\
\hline Rated power & $S$ & 526 & $\mathrm{MVA}$ \\
Rated power factor & $\cos \phi$ & $0.95(\mathrm{c})$ & - \\
AC-side voltage & $\underline{U}_{g R M S}$ & 184.75 & $\mathrm{kV}$ \\
HVDC link voltage & $U^{D C}$ & \pm 320 & $\mathrm{kV}$ \\
Phase reactor impedance & $\underline{Z}_{s}$ & $0.02+\mathrm{j} 0.1$ & $\mathrm{pu}$ \\
Arm reactor impedance & $\underline{Z}_{a}$ & $0.01+\mathrm{j} 0.08$ & $\mathrm{pu}$ \\
Converter modules per arm & $N_{u, l_{a r m}}^{k}$ & 400 & - \\
Average module voltage & $U_{S M}$ & 1.6 & $\mathrm{kV}$ \\
Sub-module capacitance & $C_{S M}$ & 8 & $\mathrm{mF}$ \\
Grid code voltage 1 & $U_{\min 1}$ & 0.9 & $\mathrm{pu}$ \\
Grid code voltage 2 & $U_{\min 2}$ & 0.6 & $\mathrm{pu}$ \\
Grid code voltage 3 & $U_{\max 1}$ & 1.05 & $\mathrm{pu}$ \\
Max reactive current & $\Delta I_{\max }$ & 1 & $\mathrm{pu}$ \\
Optimal weighting factor 1 & $\lambda_{1}$ & $10^{-9}$ & - \\
Optimal weighting factor 2 & $\lambda_{2}$ & 1 & - \\
Optimal weighting factor 3 & $\lambda_{3}$ & $10^{6}$ & - \\
Maximum MMC arm current & $I_{\max }^{\operatorname{arm}}$ & 0.77 & $\mathrm{pu}$ \\
Maximum AC grid current & $I_{\max }^{A C}$ & 1 & $\mathrm{pu}$ \\
\hline \hline
\end{tabular}

considered to be operating in the following steady-state pre-fault conditions: grid voltage $\left|U_{g}^{k}\right|=1$ pu and active and reactive power set-points $P^{k}=0.32$ pu and $Q^{k}=0$. Then, balanced and unbalanced AC voltage sags (type A, C and F) are imposed, with faulted voltage $V$ equal to $0.3 \mathrm{pu}$ [28]. The Spanish grid code is used to implement the different reference calculation strategies (see values in Table I).

Firstly, imposing the balanced AC voltage sag condition (type A [28]) results in same voltage levels for the three phases equal to $\left|U_{g}^{k}\right|=0.3 \mathrm{pu}$. Based on the grid code, as $\left|U_{g}^{k}\right|<U_{\min 2}$ (see Section IV and Table I), all phases of the AC system must receive full support from the MMC. Secondly, the unbalance type C fault (single-line to ground (SLG)) condition is imposed, leading to the following voltages $\left|U_{g}^{a}\right|=1 \mathrm{pu}$ and $\left|U_{g}^{b c}\right|=0.56 \mathrm{pu}$. Based on the grid code, phases $b$ and $c$ require 
maximum reactive current injection while phase $a \mathrm{AC}$ current should be kept constant. Finally, the unbalanced type $\mathrm{F}$ fault (line-to-line to ground (LLG)) scenario is imposed. During this event, the voltages of the three phases are $\left|U_{g}^{a}\right|=0.3 \mathrm{pu}$ and $\left|U_{g}^{b c}\right|=0.68 \mathrm{pu}$. As a result, phase $a$ requires full voltage support, whereas the remaining phases only demand partial support from the converter. Whereas the phasor form of the $\mathrm{AC}$ voltages as well as the per-phase $\mathrm{AC}$ current references are compared for each strategy and depicted in Fig. 6.

TABLE II

COMPARISON OF THE ACTIVE AND REACTIVE POWERS USING THE DIFFERENT STRATEGIES

\begin{tabular}{cccccccc}
\hline \hline \multirow{2}{*}{ Fault type } & \multirow{2}{*}{ Phase } & \multicolumn{3}{c}{$P_{g}^{k}[\mathrm{pu}]$} & & & $Q_{g}^{k}[\mathrm{pu}]$ \\
& & Opt. & Str. I & Str. II & Opt. & Str. I & Str. II \\
\hline \multirow{3}{*}{ A } & $a$ & 0 & 0 & 0 & -0.1000 & -0.1000 & -0.1000 \\
& $b$ & 0 & 0 & 0 & -0.1000 & -0.1000 & -0.1000 \\
& $c$ & 0 & 0 & 0 & -0.1000 & -0.1000 & -0.1000 \\
\hline \multirow{2}{*}{$\mathrm{C}$} & $a$ & 0.3011 & 0.1198 & 0.3167 & 0 & -0.1806 & 0 \\
& $b$ & 0 & 0.1198 & 0 & -0.0341 & -0.1806 & -0.1878 \\
& $c$ & 0.1784 & 0.1198 & 0 & -0.05864 & -0.1806 & -0.1878 \\
\hline \multirow{2}{*}{$\mathrm{F}$} & $a$ & 0.0150 & 0 & 0 & -0.0988 & -0.1778 & -0.1000 \\
& $b$ & 0.1548 & 0 & 0.1548 & -0.1659 & -0.1778 & -0.1659 \\
& $c$ & 0 & 0 & 0.1548 & -0.1659 & -0.1778 & -0.1659 \\
\hline
\end{tabular}

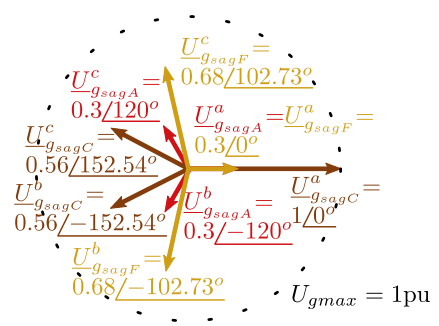

(a)

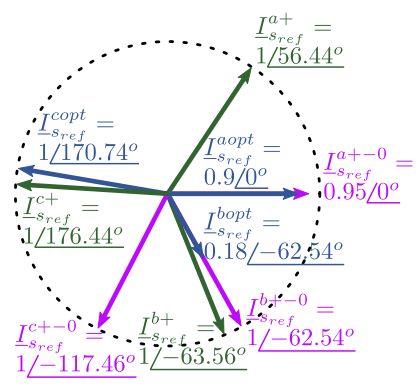

(c)

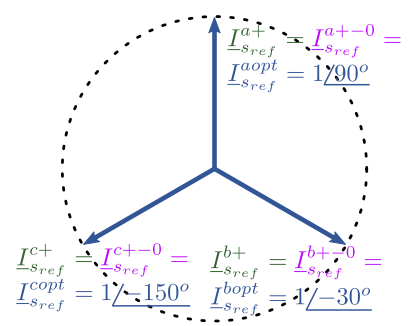

(b)

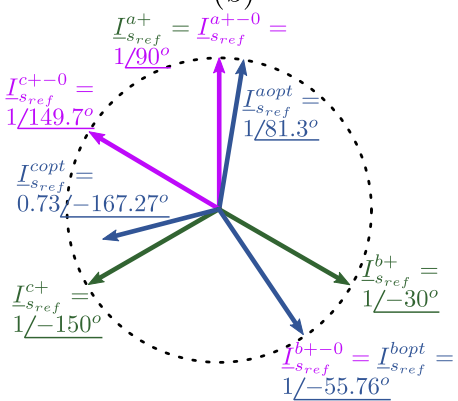

(d)

Fig. 6. Grid AC voltages and currents phasor representation for different AC grid conditions. a) Voltages for each voltage sag (A-red, C-brown, F-yellow), b) Currents for sag type A, c) Currents for sag type C and, d) Currents for sag type F. Strategy I - green. Strategy II - pink. Optimal strategy - blue.

For the different AC voltage sag conditions, shown in Fig. 6a, the output of the optimal reference calculation strategy is compared with Strategies I and II (calculated without applying optimization as detailed in Section IV). Figs. 6b to 6d depict the phasor representation of the AC grid current references obtained implementing the different strategies for the case study faults. Strategy I $\left(I_{s_{r e f}}^{k+}\right)$ is represented using green phasors, Strategy II $\left(I_{s_{r e f}}^{k+-0}\right)$ is depicted using pink phasors and the optimized current references $\left(I_{s_{r e f}}^{k o p t}\right)$ are illustrated using blue phasors. The dashed circle symbolizes the AC grid current limits $I_{\text {max }}^{A C}$. Moreover, the per-phase active and reactive powers during the faults are presented in Table II to compare the power injection performance of the different strategies.

Fig. $6 \mathrm{~b}$ and the powers presented in Table II show that the three reference calculation strategies lead to the same result for a balanced voltage sag as the grid voltages only present positive sequence component. It can be observed that all the grid currents are $90^{\circ}$ degrees phase-shifted in comparison with their phase-voltage (full voltage support), which is also in agreement with the power results as $P_{g}^{k}=0$ and $Q_{g}^{k}=0.100 \mathrm{pu}$. In terms of the optimal strategy results, the active and reactive coefficients for this case are $\alpha^{k}=1^{-5}$ and $\beta^{k}=0.999$, thus achieving a maximization of the reactive power injection. Finally, for such voltage sag condition, any of the presented strategies will completely fulfill the grid code requirements.

For fault type C, which the results are shown in Fig. 6c, it can be noticed that for the healthy phase (phase $a$ ), the optimization algorithm has a similar response to Strategy II. The obtained coefficients are $\alpha^{a}=0.951$ and $\beta^{a}=1$, meaning that phase $a$ 
current is almost not limited (it is the healthy one), and only a small reduction is applied to the active current component. To fully comply with the grid code requirements, the reactive optimal coefficient should be $\beta^{b c}=1$ for phases $b$ and $c$ to provide full reactive support, also implying that $\alpha^{b c}=0$ (no current room left for active power). However, in order to meet all the constraints imposed in the formulation of the optimization problem, such coefficients are unfeasible. Phase $b$ resulted in $\alpha^{b}=0$ and $\beta^{b}=0.18$, thus injecting no active power and only a constrained amount of reactive power. Whereas, for phase $c$, the optimization kept $\alpha^{c}=1$ and reduced $\beta^{c}=0.312$, thus providing active power and partial reactive power support. Note that, as the ideal support to be provided (Strategy II) is highly unbalanced (pink phasors in Fig. 6b), even though the optimization algorithm attempts to keep $\alpha^{k}$ and $\beta^{k}$ values as close as possible to 1 , this condition cannot be achieved due to the system and converter imposed constraints.

Further conclusions can be drawn observing the reactive and active powers injected during the voltage sag type C. As it can be noticed in Table II, the amount of active power injected for phase $a$ using the optimal algorithm is similar to the one imposed by the grid code requirement (Strategy II), whereas Strategy I leads to a larger error. Furthermore, Strategy I has high reactive power injection, which might result in undesirable over-voltages in phase $a$. For phase $b$, the active power given by the proposed optimization method also shows better performance in comparison to Strategy I. However, due to the fault characteristics and the converter constraints, the optimization algorithm cannot achieve the levels imposed by the grid operator (shown in Strategy II) for $P_{g}^{c}, Q_{g}^{b}$ and $Q_{g}^{c}$. Although the optimization is unable to fully satisfy the grid code requirement, it still is a more suitable option for such fault as it might avoid causing over-voltages in the AC system as could occur when Strategy I is employed.

For type F fault (see Fig. 6d), according to the grid code, phase $a$ requires full voltage support, whereas partial support is needed by the other phases. The optimal coefficients for phase $a$ are equal to $\alpha^{a}=0.158$ and $\beta^{a}=0.988$, indicating that the optimization algorithm presented similar response compared to Strategy II. Moreover, $\underline{I}_{s_{r e f}}^{b o p t}$ is in perfect agreement with the grid code requirement, with $\alpha^{b}=0.718$ and $\beta^{b}=1$ (partial reactive support requirement). However, it can be noted that $\underline{I}_{\text {sef }}^{\text {copt }}$ is deviated from the ideal support (Strategy II) in terms of angle and magnitude. This happens as the optimization must comply with the imposed constraints, leading to weights equal to $\alpha^{c}=0$ and $\beta^{c}=1$, which means that it is only providing reactive current support, without injecting active power (in accordance to the reactive power priorization). In summary, the optimal references obtained are able to meet the reactive power support requirements as $\beta^{k} \approx 1$ for all phases, while active power is maximized whenever possible, without violating any of the converter constraints. Finally, it can be observed in Table II that the optimization algorithm presents superior performance compared to Strategy I for all phases, as the usage of Strategy I would lead not only to undesirable over-voltages in the AC network but also to higher mismatches in the active power levels.

Next, the steady-state time-domain simulation results are analyzed once the obtained optimal references are applied to the converter model in order to verify the applicability and correctness of the model. The response of the proposed optimization algorithm (continuous lines) is compared with the waveforms of an averaged model of the MMC (dashed lines) during a type $\mathrm{C}$ fault, as shown in Fig. 7. The design limits for the arms voltage and currents, as well as, the maximum allowed value for the AC grid current and maximum and minimum equivalent arm capacitor voltage ripple are represented as dotted lines. The instantaneous powers of the upper arms, lower arms and the HVDC network are shown for all case-studies based on the results obtained both from the solution of the optimization problem and from the time-domain simulations. Moreover, the active and reactive powers exchanged with the $\mathrm{AC}$ grid are depicted in two different manners. The average active and reactive power references given by the optimization are shown as dashed orange lines, whereas the instantaneous powers are depicted as continuous magenta lines (left-bottom part). In Fig. 7, due to the unbalanced characteristic of fault, the instantaneous powers injected into the AC grid present double-line frequency oscillations. However, these oscillations are mitigated by the MMC and are not reflected into the DC network. As it can be observed, the quantities obtained from the optimization are in close agreement with the MMC averaged model, thus confirming its applicability. In addition, the AC grid currents profile match their phasorial representation (see Fig. 6c). Lastly, all the MMC quantities are within the design limits, since no waveform is surpassing its corresponding restriction, although some of them are hitting some limitations (e.g. AC current limits), due to the optimization performance and imposed constraints.

\section{B. Saturation in the MMC arm voltages}

The objective of this case study is to show the capability of the optimization method to operate with individual arm constraints. Assume that the MMC is operating in normal conditions with 400 available levels per arm (same AC voltage and power set-points as case A - pre-fault). Then, a contingency situation is imposed into the model causing a reduction of the working sub-modules in the upper arm for phase $a\left(N_{u_{a r m}}^{a}\right)$ from 400 to 330. This condition will affect the equivalent arm capacitor voltage for the upper arm in phase $a\left(U_{C_{u}}^{a}\right)$, thus limiting the voltage that can be applied with this arm. To operate in such constrained scenario, the suggested optimization formulation is used modifying only the number of available sub-modules.

Fig. 8 shows the optimization results being applied to the simulation model in such case. It can be seen that the stored energy (voltage) in phase $a$ is importantly reduced (see Fig. 8 top-left graph), impacting both its DC and AC voltage levels. In order to avoid over-modulation, the applied voltages in the upper arm of phase $a$ are decreased and this also affects the 


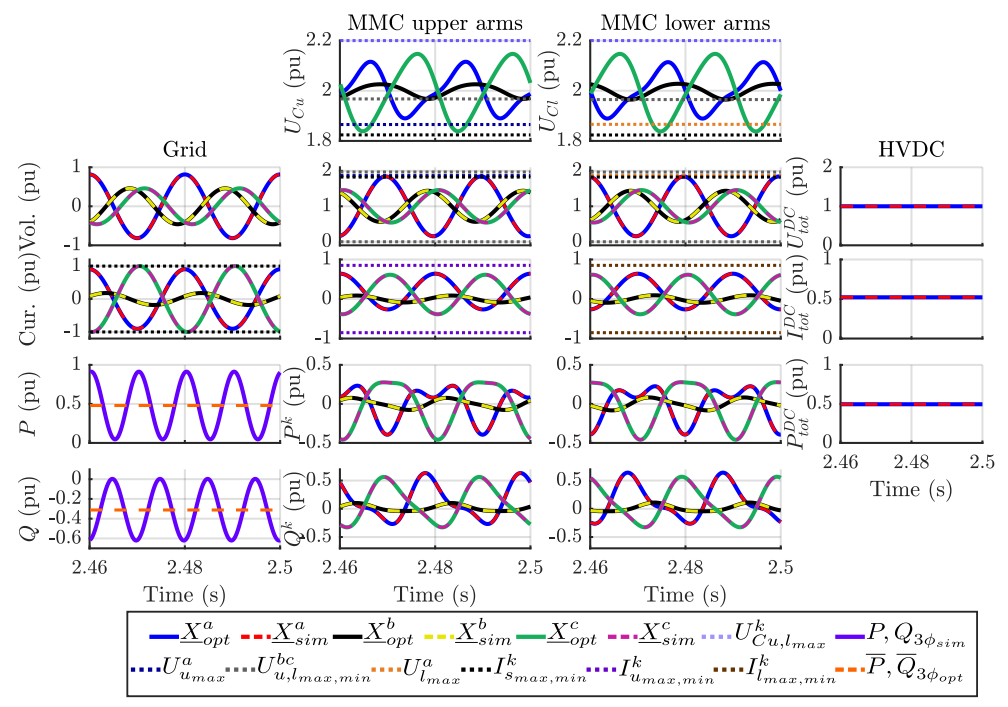

Fig. 7. Steady-state waveforms of the MMC quantities during a type $\mathrm{C}$ fault.

applied voltage for the lower one, which must be adapted to fulfill the optimization constraints. Furthermore, phases $b$ and $c$ applied voltages are increased (to compensate the reduction in phase $a$ ) by the optimization algorithm targeting to meet the pre-contingency active and reactive power set-points. These voltages are incremented without entering over-modulation mode, as the optimization is limiting $\underline{U}_{u, l}^{b c}$ quantities to their respective maximum levels.

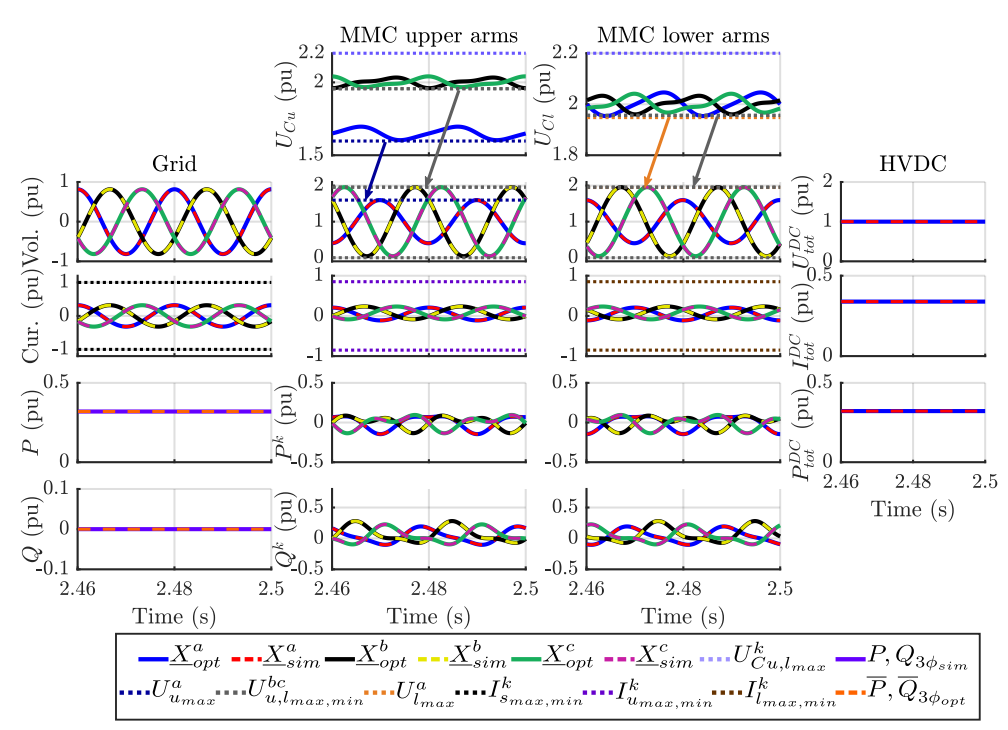

Fig. 8. MMC waveforms during constraint scenario for the arms voltages.

Even though the optimization attempts to achieve the pre-fault power set-point values, the converter limitations and constraints do not allow the MMC to reach the desired power levels, consequently the power flow through the MMC is reduced. This is reflected in the optimization coefficients setting them to $\alpha^{k}=0.364$ and $\beta^{k}=1$ (equal power/current per phase), which is constraining the active power injection to the AC side. In addition, although there is energy imbalance inside the converter, the optimization is capable of attaining steady-state conditions by establishing an internal power flow among the MMC phases (see Fig. 8 arm currents).

\section{Maximum capacitor voltage fluctuation}

This case study aims to demonstrate that the optimization is able to limit the maximum equivalent arms' capacitor voltages based on the estimation derived in Section III-E. Considering that the MMC is again operated under pre-fault defined settings, two different maximum voltage ripple scenarios are applied to all the converter's arms. The optimization results for the two cases are shown in Fig. 9. For the first condition (see Fig. 9a), the maximum voltage ripple for all arms is equal to $10 \%$ of the arm voltage reference $\left(N_{u, l_{a r m}}^{k} \cdot U_{S M}=640 \mathrm{kV}\right)$, which represents the settings used in the previous case studies. Whereas, Fig. $9 \mathrm{~b}$ displays the waveforms when the maximum ripple is reduced to $5 \%$. 
In the first case, the value of the maximum ripple is higher than the operating voltage stored in the equivalent arms' capacitors (see Fig. 9a), keeping a safety margin. Thus, the optimization coefficients are equal to $\alpha^{k}=1$ and $\beta^{k}=1$ (not limiting any power). However, if the maximum voltage limitation is reduced (see Fig. 9b), the optimization decreases the active power exchanged by the converter $\left(\alpha^{k}=0.74\right.$ and $\left.\beta^{k}=1\right)$ in order to maintain the converter within its acceptable limits. The reduction in the amount of active power injected into the AC system happens due to the necessity of decreasing the stored energy magnitudes in the equivalent arm capacitors to avoid exceeding the design limitation. Otherwise, there is no possible alternative to limit the equivalent arm capacitor voltage ripple to the imposed level, which is in agreement with (16) and (17). Also, it can be confirmed that the ripple estimation used in the optimization is valid, as the maximum and minimum equivalent arm capacitor voltages are kept within the bounds imposed via (19) (see the top graphs of Fig. 9b). Finally, the errors for the maximum and minimum equivalent arm capacitor voltage between the actual value (obtained based on the analytical expression regarding the first and second order frequency terms (15)) and the maximum and minimum bounds (19) are: $e_{\max }=0.5 \%$ and $e_{\min }=1.34 \%$ considering maximum allowed voltage ripple equals to $10 \%$, whereas $e_{\max }=0.46 \%$ and $e_{\min }=1 \%$ when the maximum allowed ripple is reduced to $5 \%$.

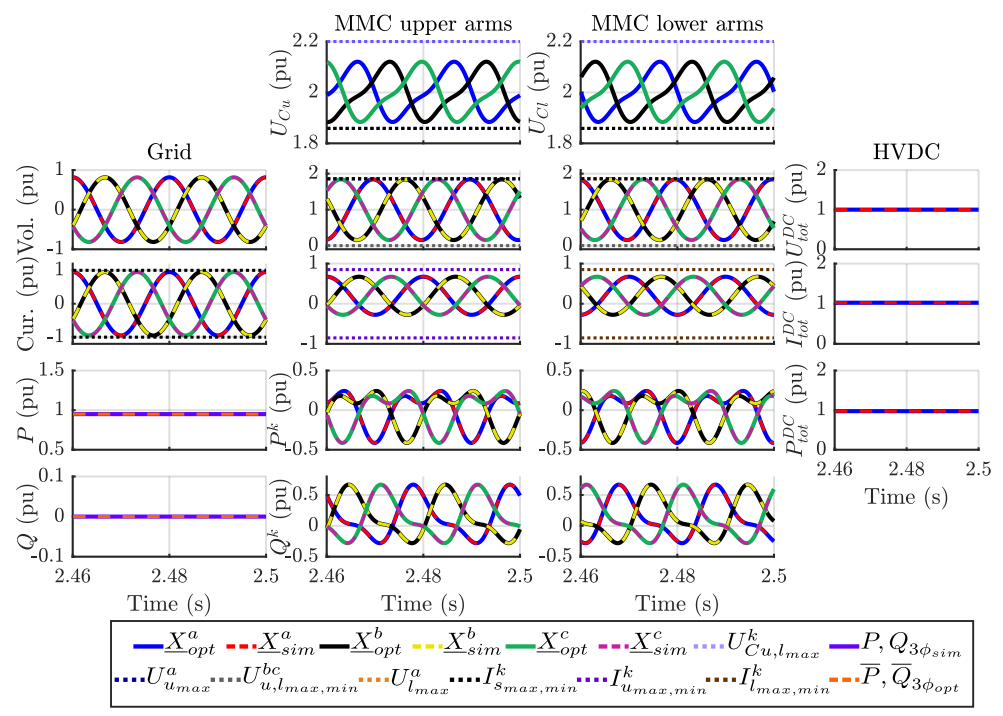

(a) Maximum ripple equals to $10 \%$.

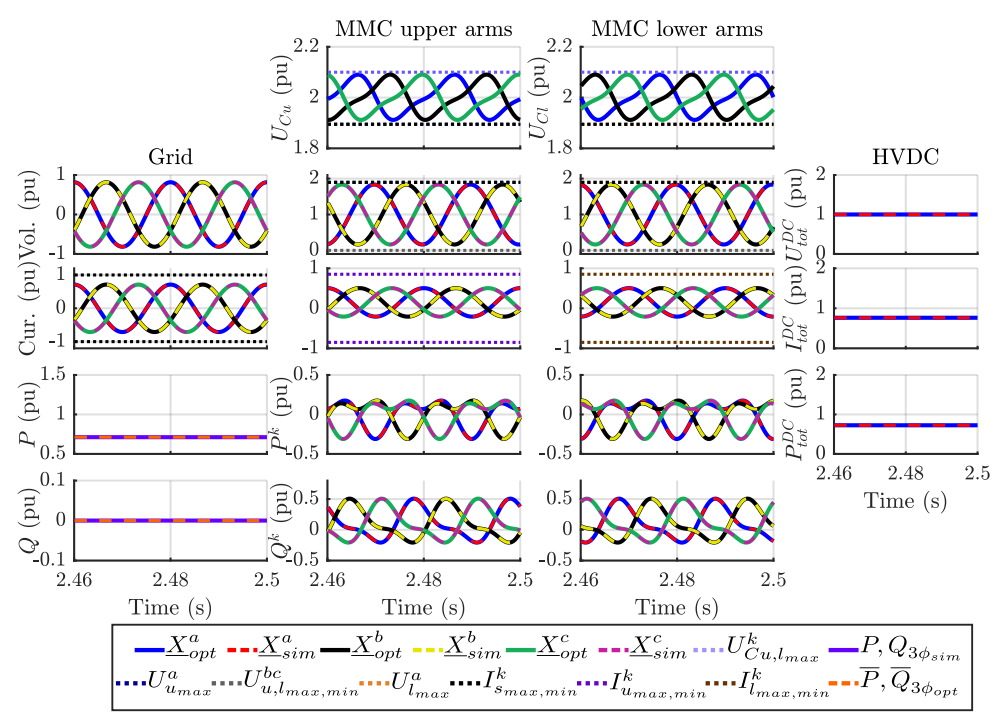

(b) Maximum ripple equals to $5 \%$.

Fig. 9. Time-domain waveforms for the equivalent arms' capacitor voltages. a) Maximum ripple equals to $10 \%$ and, b) Maximum ripple equals to $5 \%$.

\section{CONCLUSION}

An optimization-based reference calculation method for MMCs operating in normal and constrained situations (converter variables limited due to voltage or current limitations) has been presented. The optimization problem has been formulated based 
on the steady-state per arm approach model, which permits analyzing individually each converter arm while enabling to impose specific arm constraints. The limitations considered have been each individual arm currents and voltages and the sub-module capacitor voltages, in which the last one was obtained through an analytic estimation. In addition, the optimization algorithm has been formulated as a multi-objective problem, allowing to prioritize between active power, reactive power or converter arm losses reduction when the converter is operating in a constrained condition. Results show that the optimization algorithm is able to provide adequate converter references in different scenarios, such as unbalanced fault conditions, reduction of the arm available sub-modules or limited arm ripple operation. The optimization output references are validated using time-domain simulations, which in all cases revealed the correct operation of the converter, attempting to fulfil the grid code requirements while maintaining the converter within the defined voltage and current limits.

\section{REFERENCES}

[1] D. Van Hertem, O. Gomis-Bellmunt, and J. Liang, HVDC Grids: For Offshore and Supergrid of the Future, ser. IEEE Press Series on Power Engineering. Wiley, 2016.

[2] E. Prieto-Araujo, A. Junyent-Ferré, G. Clariana-Colet, and O. Gomis-Bellmunt, "Control of modular multilevel converters under singular unbalanced voltage conditions with equal positive and negative sequence components," IEEE Trans. Power Syst., vol. 32, pp. 2131-2141, May 2017.

[3] E. Sánchez-Sánchez, E. Prieto-Araujo, A. Junyent-Ferré, and O. Gomis-Bellmunt, "Analysis of MMC energy-based control structures for VSC-HVDC links," IEEE Trans. Emerg. Sel. Topics Power Electron., vol. 6, no. 3, pp. 1065-1076, Sep. 2018.

[4] A. Lesnicar and R. Marquardt, "An innovative modular multilevel converter topology suitable for a wide power range," in 2003 IEEE Bologna Power Tech Conf. Proc., vol. 3, June 2003, p. 6 pp.

[5] S. Rohner, S. Bernet, M. Hiller, and R. Sommer, "Modulation, losses, and semiconductor requirements of modular multilevel converters," IEEE Trans. Ind. Electron., vol. 57, no. 8, pp. 2633-2642, Aug 2010.

[6] J. Peralta, H. Saad, S. Dennetiere, J. Mahseredjian, and S. Nguefeu, "Detailed and averaged models for a 401-level MMC-HVDC system," IEEE Trans. Power Del., vol. 27, no. 3, pp. 1501-1508, July 2012.

[7] E. Prieto-Araujo, A. Junyent-Ferré, C. Collados-Rodríguez, G. Clariana-Colet, and O. Gomis-Bellmunt, "Control design of modular multilevel converters in normal and AC fault conditions for HVDC grids," Electr. Pow. Syst. Res., vol. 152, pp. 424 - 437, 2017.

[8] X. Li, Q. Song, W. Liu, S. Xu, Z. Zhu, and X. Li, "Performance analysis and optimization of circulating current control for modular multilevel converter," IEEE Trans. Ind. Electron., vol. 63, pp. 716-727, Feb 2016.

[9] Q. Song, W. Liu, X. Li, H. Rao, S. Xu, and L. Li, "A steady-state analysis method for a modular multilevel converter," IEEE Trans. Power Electron., vol. 28, no. 8, pp. 3702-3713, 2013.

[10] R. Oliveira and A. Yazdani, "An enhanced steady-state model and capacitor sizing method for modular multilevel converters for HVDC applications," IEEE Trans. Power Electron., vol. 33, pp. 4756-4771, June 2018.

[11] O. C. Sakinci and J. Beerten, "Generalized dynamic phasor modeling of the MMC for small-signal stability analysis," IEEE Trans. Power Del., vol. 34, no. 3, pp. 991-1000, June 2019.

[12] M. Guan and Z. Xu, "Modeling and control of a modular multilevel converter-based HVDC system under unbalanced grid conditions," IEEE Trans. Power Electron., vol. 27, no. 12, pp. 4858-4867, Dec 2012

[13] J. Wang, J. Liang, C. Wang, and X. Dong, "Circulating current suppression for MMC-HVDC under unbalanced grid conditions," IEEE Trans. Ind. Appl., vol. 53, no. 4, pp. 3250-3259, July 2017.

[14] Y. Liang, J. Liu, T. Zhang, and Q. Yang, "Arm current control strategy for MMC-HVDC under unbalanced conditions," IEEE Trans. Power Del., vol. 32, no. 1, pp. 125-134, Feb 2017.

[15] Z. Ou, G. Wang, and L. Zhang, "Modular multilevel converter control strategy based on arm current control under unbalanced grid condition," IEEE Trans. Power Electron., vol. 33, no. 5, pp. 3826-3836, May 2018.

[16] G. Bergna-Diaz, J. A. Suul, E. Berne, J. Vannier, and M. Molinas, "Optimal shaping of the MMC circulating currents for preventing AC-side power oscillations from propagating into HVDC grids," IEEE Trans. Emerg. Sel. Topics Power Electron., vol. 7, pp. 1015-1030, June 2019.

[17] X. Shi, Z. Wang, B. Liu, Y. Li, L. M. Tolbert, and F. Wang, "Steady-state modeling of modular multilevel converter under unbalanced grid conditions," IEEE Trans. Power Electron., vol. 32, no. 9, pp. 7306-7324, Sep. 2017.

[18] X. Shi, Z. Wang, B. Liu, Y. Liu, L. M. Tolbert, and F. Wang, "Characteristic investigation and control of a modular multilevel converter-based HVDC system under single-line-to-ground fault conditions," IEEE Trans. on Power Electron., vol. 30, no. 1, pp. 408-421, Jan 2015.

[19] National Grid TSO, The Grid Code, Sept 2019.

[20] Elia, Proposal for NC HVDC requirements of general application, May 2018.

[21] Red Elétrica de España, Procedimiento de Operación 12.4: Requisitos Técnicos Mínimos de Conexión de Sistemas HVDC y Módulos de Parque Eléctrico Conectados en Corriente Continua, Jun 2018.

[22] K. Sharifabadi, L. Harnefors, H. P. Nee, S. Norrga, and R. Teodorescu, Design, Control and Application of Modular Multilevel Converters for HVDC Transmission Systems. Wiley-IEEE press, 2016.

[23] M. Tapia, "Use of superposition in writing state equations for networks with excess elements," IEEE Trans. Circuit Theory, vol. 17, no. 4, pp. 624-626, Nov 1970.

[24] D. W. Spier, E. Prieto-Araujo, O. Gomis-Bellmunt, and J. López-Mestre, "Steady-state analysis of the modular multilevel converter," in 45th Annu. Conf. of the IEEE Ind. Electron. Soc., Oct 2019, p. 6 pp.

[25] H. Saad, S. Dennetiére, J. Mahseredjian, P. Delarue, X. Guillaud, J. Peralta, and S. Nguefeu, "Modular multilevel converter models for electromagnetic transients," IEEE Trans. Power Del., vol. 29, pp. 1481-1489, June 2014.

[26] D. W. Spier, E. Prieto-Araujo, O. Gomis-Bellmunt, and J. López-Mestre, "Analytic estimation of the MMC sub-module capacitor voltage ripple for balanced and unbalanced AC grid conditions," in $3^{\circ}$ Simpósio Ibero-Americano em Microrredes Inteligentes com Integração de Energias Renováveis, Oct 2019, p. 6 pp.

[27] J. Wang, J. Liang, F. Gao, X. Dong, C. Wang, and B. Zhao, “A closed-loop time-domain analysis method for modular multilevel converter," IEEE Trans. Power Electron., vol. 32, no. 10, pp. 7494-7508, 2017.

[28] M. Bollen and L. Zhang, "Different methods for classification of three-phase unbalanced voltage dips due to faults," Electr. Power Syst. Res., vol. 66, no. 1, pp. $59-69,2003$.

[29] Z. Ali, N. Christofides, L. Hadjidemetriou, E. Kyriakides, Y. Yang, and F. Blaabjerg, "Three-phase phase-locked loop synchronization algorithms for gridconnected renewable energy systems: A review," Renew. Sust. Energ. Rev., vol. 90, pp. 434 - 452, 2018.

[30] S. Kim, K. Koh, M. Lustig, S. Boyd, and D. Gorinevsky, "An interior-point method for large-scale $\ell_{1}$-regularized least squares," IEEE J. Sel. Topics Signal Process., vol. 1, no. 4, pp. 606-617, Dec 2007. 